\title{
A aurora do (forte)piano
}

\author{
Pedro Persone (UNESP/ FAPESP, São Paulo) \\ pedro@persone.net; http://fortepiano.org
}

\begin{abstract}
Resumo. Este artigo revisita a aurora do fortepiano, instrumento que se tornaria ponto de convergência no fazer musical de fins do século XVIII até meados do XX, e apresenta referências documentais sobre o início da invenção da mecânica de Bartolomeo Cristofori, bem como documentações de outros projetos não Cristoforianos.

Palavras-chave: Piano, Fortepiano, Cristofori, Tangentflügel, Piano tangente.
\end{abstract}

\section{The dawn of the (forte)piano}

Abstract. This article reviews the dawn of the fortepiano, an instrument central to music making from the second half of Eighteenth century to the middle of the Twentieth century. It presents documents that describe the beginning of Cristofori's invention as well as non-Cristofori documents on the same subject.

Keywords: Piano, Fortepiano, Cristofori, Tangentflügel, Tangent piano.

\section{1- Os princípios de um novo instrumento}

Antes do advento do registro de patentes, muitas vezes poderia ser difícil conectar a invenção ao seu criador - a idéia da patente é mais comum ao século XIX. Com a criação da lei de patentes, por volta de 1790 nos Estados Unidos, toda nova invenção, mudança, ou aprimoramento podiam ser diretamente relacionados a seu inventor ou executor. No caso particular do piano, podemos traçar suas origens e desenvolvimentos, sendo possivel determinar a provável data, o lugar e seu inventor. É possível ver exemplos de melhorias patenteadas posteriormente na história do piano por Erard ou Pleyel. Cada invenção ou criação materializada por um construtor nas diversas etapas da história do fortepiano parece ter sido inspirada pelo desejo de fazer um instrumento de teclado que permitisse ao intérprete tocar expressivamente com douceur, forte e piano. ${ }^{1}$

Desde o aparecimento, na Itália em 1711, do célebre artigo de Scipione MAFFEI (1675-1755) (MAFFEI, 1711, p.144-159), posteriormente traduzido para o alemão (MATTHESON, 1722-1725, p.336-343), até as mais recentes pesquisas, Bartolomeo Cristofori (16551731) pode ser considerado o inventor do (forte)piano. Examinando a história do instrumento podemos aprender que Henry Arnaut de Zwolle (fins do século XIV-1466), em um manuscrito de ca. 1440, descreve um clavisimbalum - instrumento semelhante ao cravo - com uma ação de martelos. Hippolito Cricca, em três dentre as cartas que escreveu ao duque Cesare d'Este (1561-1628), de Modena, no ano de 1598, menciona l'instromento pian et forte con l'orghano, ou istromento piane e' forte, ou ainda instromento piano et forte (POLLENS, 1995, p.229-231). Cricca não descreveu claramente este "instrumento suave e forte", assim não podemos ter uma idéia real sobre o mecanismo usado. Mas está claro que tal instrumento havia sido utilizado na corte dos $d^{\prime} E s t e$, em Ferrara. 0 mais antigo instrumento conhecido, um "octave virginal" ou espineta construído em 1587 por Franciscus Bonafinis, teve sua mecânica original substituída por uma mecânica a martelos tangentes em 1632.

Jean Marius (?-1720), um hábil matemático, com graduação em leis, inventor do clavecin-brisé (cravo dobrável), submeteu em 1716 à Académie Royale des Sciences de Paris quatro diferentes projetos de clavecin $\dot{a}$ maillets (cravo a martelos): um tinha martelos (maillets) substituindo os saltarelos, o segundo uma combinação de martelos e saltarelos que poderiam ser usados em separado ou em conjunto; um terceiro incluindo duas maneiras de golpear as cordas, uma por baixo e outra por cima; e um quarto projeto que era um modelo de "armário". Nenhum destes instrumentos chegou a ser construido (POLLENS, 1995, p.265-267).

Na Saxônia, Christoph Gottlieb Schröter (1699-1782) apresentou uma idéia similar em 1717. Mais uma vez nenhum instrumento foi construído. Além disso, Marius e Schröter estavam experimentando com uma mecânica mais direcionada a um piano tangente (Ex.1). Excelentes textos de Pollens (POLLENS, 2000, p.140-151) sobre as correspondências entre Schröter e Friedrich Wilhelm Marpurg (1718-1795) apresentam tradução do documento para o inglês. 0 documento de Schröter sobre sua auto-proclamada invenção aparece primeiramente no livro de Lorenz Christoph Mizler (1711-1778), Neu eröffnete musikalische Bibliothek publicado em Leipzig entre os anos de 1739 e 1754 - vol. 3, pt. 3 - (POLLENS, 1995, p. 244-245) e, posteriormente, em 1763, sua carta foi publicada no livro Kritische Briefe über die Tonkunst. Berlin, 1760-64, vol. 3, pt. 1, de Friedrich Wilhelm Marpurg (1718-1795) (POLLENS, 1995, p. 246-264). 
A carta de Schröter (1738), constante na Neu eröffnete de Mizler, explica que em 1717 foi comissionado a ele um instrumento novo de teclado usando martelos. No Kritische de Marpurg, Schröter explicou que seu modelo - uma mecânica acionada golpeando a corda tanto por baixo quanto por cima - fora submetido à apreciação da corte em Dresden. Dessa corte, Schröter nunca obteve nenhum resultado sobre uma possivel aprovação ou mesmo desaprovação. Além desse silêncio, os documentos originais encaminhados à corte nunca foram devolvidos ao inventor. Não sabemos se algum instrumento de Schröter alcançou um estágio de construção real, uma vez que nenhum sobreviveu. Schröter projetou uma ação totalmente diferente daquela de Cristofori. Provavelmente ignorava a data do Arpicimbalo de Cristofori. Se não há indícios da existência real de nenhum instrumento de
Marius ou de Schröter, podemos entender que a linha mais importante - não Cristoforiana - de instrumentos a martelos é a do tangent piano.

No Tangentenflügel (Ex.2), as peças de madeira (tangentes) deslizam livremente para golpearem as cordas (letra C no Ex.2). Estas peças de madeira assemelhamse mais a saltarelos do cravo que a martelos articulados ou pivotados, tal como na invenção de Cristofori. Franz Jakob Späth (1714-1786), de Regensburg, inventou, em 1751, um dos instrumentos tangentes mais importantes. Alguns dos princípios mecânicos do piano tangente foram incorporados em grande número de projetos. Atualmente, em Ninove, Bélgica, um construtor de clavicórdios e fortepianos, Ghislain Potvlieghe - que gentilmente cedeu o esboço da mecânica acima - constrói cópias dos Tangentenflügel de Baldassare Pastore, 1799.
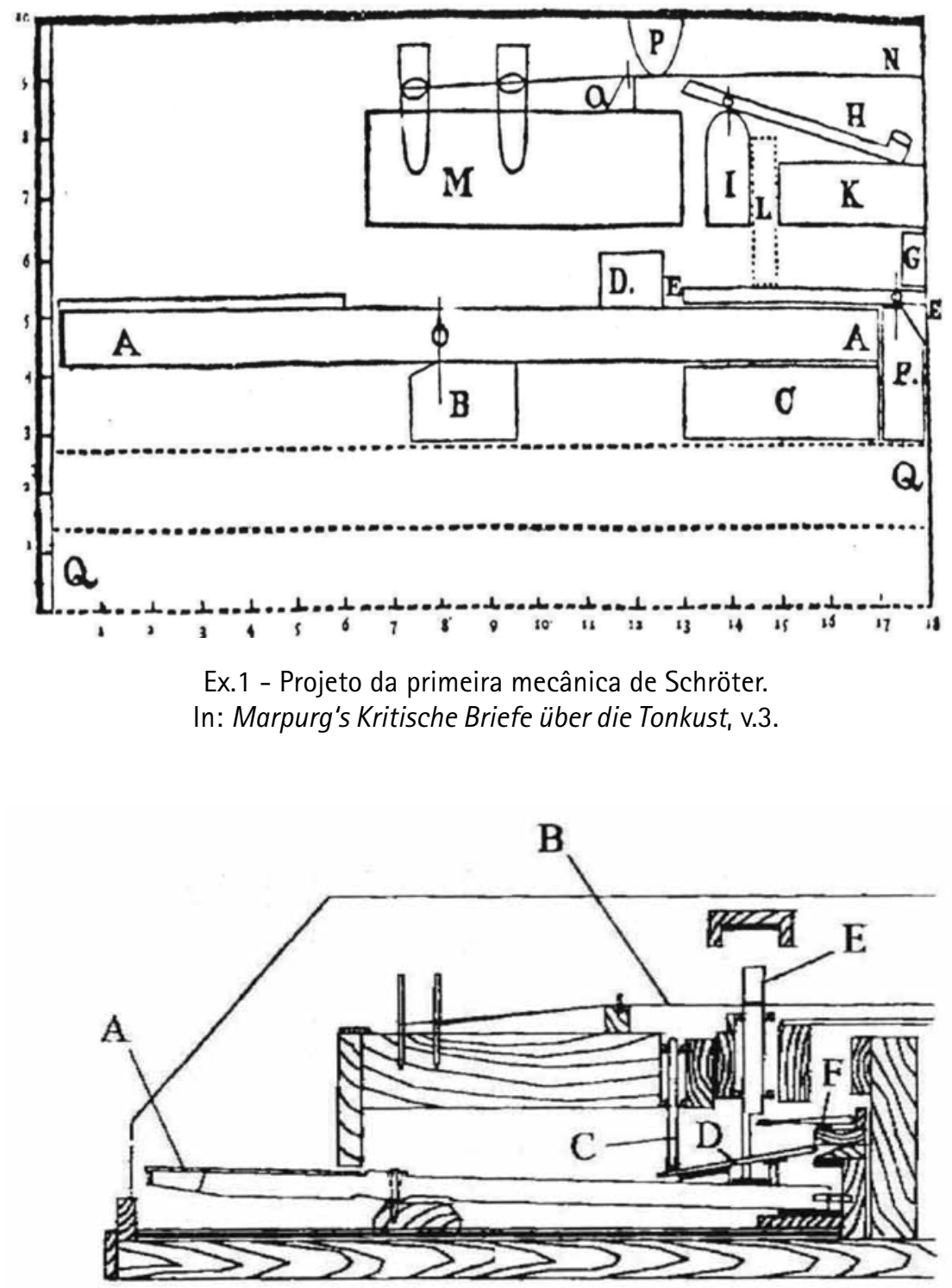

The tangent action

A: key B: string C: tangent D: accelerating lever E: damper F: damper litter

Ex.2 - Design da mecânica tangente por Ghislain Potvlieghe (Ninove, Belgium) ${ }^{2}$ 
Outro alemão, Pantaleon Hebenstreit (1668-1750), o mais famoso virtuose de dulcimer associou-se a Gottfried Silbermann (1683-1753) de Freiberg, que seguiu as primeiras instruções para a construção de um dulcimer martelado não mecânico que se tornou conhecido como Pantaleon (Ex.3). Em 1697 Hebenstreit fez um novo modelo de dulcimer ${ }^{3}$ combinando cordas de tripa e metal juntas. Este grande instrumento media mais de 9 pés. $A$ técnica de Hebenstreit alcançou um nível tão elevado de perfeição que se tornou quase impossivel imitar o seu toque. Hebenstreit tocava seu Pantaleon com martelos de madeira alterados às vezes por outros cobertos por material macio. Como o instrumento não tinha abafadores, as cordas vibravam livremente, tendo por resultado um som extremamente rico e poderoso.

Sua habilidadecomo pantaleonista (eviolinista)foielogiada por Georg Philipp Telemann (1681-1767) e por Johann Kuhnau (1660-1722), Cantor da Igreja de São Thomas em Leipzig. Kuhnau definiu a execução ao pantaleon como um "trabalho de Hercules", devido à grande dificuldade de se tocar tal instrumento. Esta sonoridade maravilhosa provavelmente motivou a adaptação de um teclado. $\mathrm{Na}$ correspondência de Hebenstreit com Telemann é mencionada outra invenção de Silbermann, o Cembal $d^{\prime}$ Amour, um pequeno instrumento de quatro oitavas com tampo harmônico duplo associando a funcionalidade do cravo ao som delicado do clavicórdio.

Equipado com um teclado e uma mecânica simples de martelos com alguns registros, antigos dulcimers com teclado poderiam, então, imitar o toque de Hebenstreit. Além do som brilhante normal, os registros eram os seguintes:
- moderador, efeito resultante da introdução de feltro ou couro entre as cordas e os martelos ou tangentes;

- registro de alaúde (similar ao registro de alaúde do cravo) utilização de cordas de tripa ou superfície macia no martelo ou no tangente;

- damper pedal ${ }^{4}$ um pedal de abafador (ou alavanca de abafador do joelho) que tinha o efeito de tirar os abafadores das cordas que, então, podiam vibrar livremente da mesma maneira que o dulcimer sem abafadores.

Com base na existência da espineta de Bonafinis e de sua ação de tangentes, os detratores modernos da primazia de Cristofori, indicam que o piano teve uma longa (pré) história anterior a Bartolomeo Cristofori. Devido à falta de instrumentos sobreviventes, o conhecimento de pianos não-Cristoforianos ou pré-Cristoforianos torna-se uma matéria muito difícil e praticamente especulativa.

Baseando sua teoria em um único exemplo - a espineta de Bonafis - e também em documentos originais préCristofori, os detratores da paternidade cristoforiana do piano supõem a existência e, provavelmente, alguma construção de instrumentos similares ao piano. De fato, não se pode negar o conhecimento de mecânicas a martelos como os mencionados por originais de fontes preliminares - tais como de Zwolle e Cricca, mencionados acima - mas não podemos supor nenhuma manufatura de tais instrumentos. De acordo com tal pensamento, Cristofori pode ser considerado uma figura importante no desenvolvimento do piano, mas não pode ser considerado o ponto inicial de sua história. Os detratores da invenção

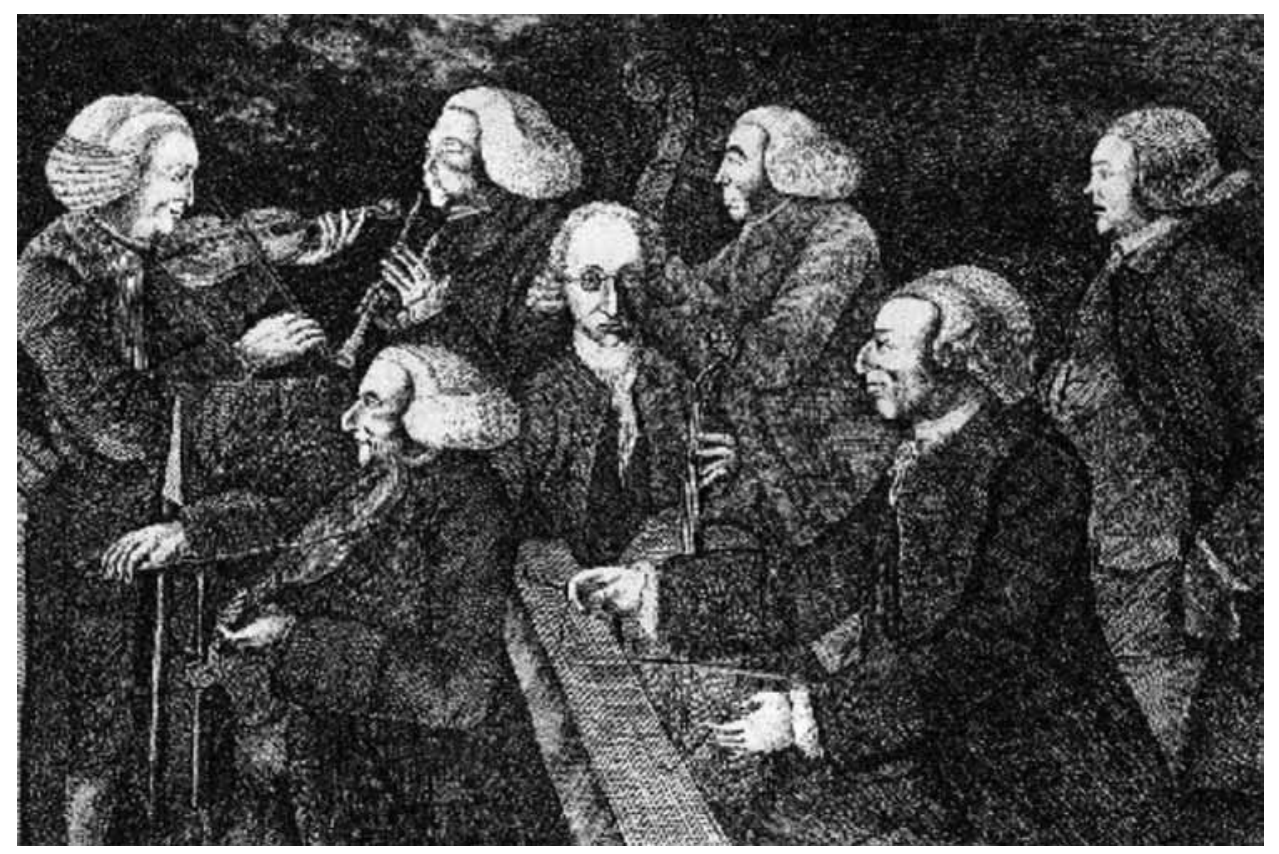

Ex. 3 - Concerto na Christ's College, Cambridge, Junho de 1767: gravura de Abraham Hume segundo Thomas Orde mostrando (da esquerda à direita) P. Hellendaal (violino), V. Noel (violino), J.F. Ranish (oboé), West (violoncello), J. Wynne, compositor e editor de música em Cambridge (contrabaixo), G. Noel (Pantaleon) e D. Wood (cantor e posteriormente organista na Ely Cathedral). 
cristoforiana acreditam que o fato de ter as cordas golpeadas por um martelo pivotado ou por tangentes de madeira de movimento livre não importa, desde que, como resultado final, se consiga um efeito similar. Os pianos tangentes tiveram uma idade de ouro entre os anos de 1750 e de 1830, quando este tipo de piano foi amplamente usado na Europa.

0 piano tangente foi um sucesso no objetivo principal de manter a sonoridade rica, leve e brilhante do cravo, tendo a possibilidade de nuances dinâmicas. A sonoridade e o toque do piano tangente demonstram qualidades distantes das características do fortepiano tais como os instrumentos dos anos 1780/90. Acreditam também que Pantaleon Hebenstreit desempenhou um papel decisivo - mesmo que contra sua própria vontade - no desenvolvimento do piano.

\section{2 - Bartolomeo Cristofori (1655-1732), inventor ou estruturador?}

Bartolomeo Cristofori nasceu em 4 de Maio de 1655 na cidade de Pádua, República de Veneza e morreu em Florença em 27 de Janeiro de 1732. Brunelli Bonetti transcreveu a certidão de batismo da paróquia de San Lucca (BRUNELLI BONETTI, 1955, p. 31):

\footnotetext{
No dia 6 de Maio de 1655, Bartolomio, filho de Francesco Christofani e de Laura sua esposa, foi batizado por mim, Don Gagliardi, seu pároco, presentes à Santa Fonte o Sr. Camillo Chinoni da paróquia de San Lorenzo. A madrinha foi Pani Lina, empregada da Sra. Laura Papafava da paróquia do Duomo, nasceu no dia 4 do presente mês as 6:00 horas da manhã. ${ }^{5}$
}

Devido a uma extensiva documentação (quase de forma compulsiva) dos registros mantidos pela dinastia dos Medici, temos uma rica fonte de informação sobre Cristofori.

Após haver examinado alguns dos instrumentos de Cristofori em 1687, o Grão Duque Ferdinando de' Medici (1663-1713) convenceu Cristofori (Ex.4) a fixar residência em Florença, onde sua perícia se mostraria um grande beneficio à corte dos Medici, mas não se sabe ao certo quais as circunstâncias dessa reunião. 0 cembalaro ${ }^{6}$ Cristofori, então com trinta e três anos de idade, não se mostrou entusiasmado a princípio, ${ }^{7}$ finalmente aceitou a oferta (O'BRIEN, 1994, p.69). Cristofori sempre teve a intenção de retornar a sua Pádua natal, como podemos ver em seu testamento, nunca tendo alcançado esse intento.

Alguns autores acreditam que as famílias Papafava, de Pádua, ou Albizi, de Florença, iniciaram as apresentações entre Ferdinando e Cristofori. Ferdinando tinha razões práticas para empregar alguém como Cristofori. Em 1700 Ferdinando possuía pelo menos trinta e três cravos, dois clavicórdios e dois órgãos para contínuo.

Embora experiente e com ótima reputação como construtor de cravos, $^{8}$ podemos considerar a carreira de Cristofori a partir deste ponto. 0 príncipe Ferdinando apontou-o para o grupo de músicos da corte - conhecido como virtuosi ${ }^{9}$ - e pagou-lhe um salário mensal, bem como o aluguel de seu atelier na via a canto agli Alberti (conhecida hoje como Via de' Neri) onde mantinha dois assistentes, Giovanni Ferrini (?1669-1758) e outro não citado nas fontes.

0 Grão Príncipe Ferdinando, filho de Cosimo III (16421723), focou mais seus interesses em música e arte que em política. Ferdinando alcançou uma excelente reputação como hábil cravista, que podia ler e memorizar à primeira vista. Cultivou a ópera, a música instrumental e a commedia dell'arte em sua corte. Na lista de sua equipe de funcionários e dos virtuosi podemos ler os nomes de doze indivíduos, onze performers e um strumentaio (O'BRIEN, 1994, p. 55):

- Francesco de Castris
- Raffaelo Baldi
- Martino Bitti
- Domenico Salvetti
- Lodovico Ermano
- Niccolò Sussier
- Alessandro Bitti
- Antonio Francesco Carli
- Francesco Mannucci
- C.Maggi
- Carlo Casali
- Bartolomeo Cristofori.

Cristofori, um homem de mente inquiridora e de engenhosa imaginação técnica, experimentou com mecanismos de teclado e produziu tipos especiais de instrumentos. 0 Arpicimbalo ou 'gravicembalo col piano e forte', significando um instrumento capaz de gradações dinâmicas, ${ }^{10}$ - deve ser considerado apenas uma dentre suas invenções. De fato, foi esse Arpicimbalo que o projetaria para as gerações futuras e que desempenhou um papel decisivo na história dos instrumentos de teclado.

A referência mais antiga conhecida a um piano de Cristofori pode ser encontrada no inventário anônimo dos Medici em 1700.11

Um Arpicembalo recém inventado por Bartolomeo Cristofori, que produz o fraco e forte, com dois jogos de cordas em unissono, com o tampo harmônico de cipreste sem rosácea, com lateral e moldura parcialmente torneada com tiras de ébano, com alguns saltarelos com pano vermelho [abafadores] que tocam nas cordas e alguns martelos que produzem o piano e forte, e todo o mecanismo é coberto por uma folha de madeira do cipreste filetada com ébano, com teclas em bucho e ébano sem rachaduras que começam no cisolfaut na oitava do baixo e terminam no cisolfaut com 49 teclas branco e preto, com os dois blocos negros nas extremidades para elevar e obstruir [o teclado] com dois botões pretos acima, com o comprimento três braccia e sete oitavos [217.6cm], a largura frontal de um braccio e seis soldi [98.6cm], com sua estante da música da madeira de cipreste, e sua contra caixa externa de madeira branca, e de sua tampa de couro vermelho forrada com tafetá verde e orlada com fita de ouro. ${ }^{12}$

Cristofori foi o único a construir instrumentos de teclado a martelos reais e tocáveis, além dos pianos tangentes 
que viriam posteriormente. 0 texto acima, de uma fonte primária: Inventário de diversos tipos de instrumentos musicais de propriedade do Sereníssimo Príncipe Ferdinando da Toscana, em um manuscrito encadernado datado de 1700, descreve o Arpicembalo recentemente inventado ou clavicembalo col piano e forte. Não sabemos o nome do autor do inventário, devido à falta da página de título no manuscrito, mas reconhecemos esta como sendo a fonte mais antiga e segura a fixar a data do fortepiano de Cristofori. Podemos, assim, inferir que o arpicimbalo veio à luz por volta do ano de 1700 (O'BRIEN, 1994,p. 103-110).

Os pesquisadores se sentem completamente seguros ao afirmar que Cristofori teve a idéia de desenvolver uma mecânica a martelos (Ex.5) antes de sua mudança para Florença. Nas anotações inéditas feitas por Scipione Maffei (1675-1755) ${ }^{13}$ a fim escrever seu famoso artigo para o quinto volume do Giornale de' Letterati d'Italia 14 sobre a nova invenção, podemos ler que Cristofori a desenvolveu por seu próprio desejo, o que contradiz diretamente a idéia geral de que houve algum tipo de instigação pessoal por parte de Ferdinando. Este detalhe deve ter sido intencionalmente omitido no artigo arcadiano de Maffei, que intencionalmente quis elogiar Ferdinando de alguma maneira. Podemos encontrar em suas notas: Bortolo Cristofali de Pádua, um empregado do Príncipe, inventou um cravo com piano e forte por sua própria vontade, e até o momento construiu três, dois vendidos em Florença e um ao Cardeal Ottoboni. ${ }^{15}$
0 Cardeal, ${ }^{16}$ em 1709, escreveu uma carta ao Príncipe para agradecer o raro cravo com que Ferdinando o favoreceu. Cristofori permaneceu em Florença depois da morte de Ferdinando e se tornou o curador (custode) da coleção de instrumentos musicais dos Medici até sua morte em 1732 (O'BRIEN, 1994, p.90) ${ }^{17}$

Cristofori deve ser considerado o "inventor" do fortepiano (não apenas mais um experimento, mas de um verdadeiro sucesso em funcionamento), apesar das "reivindicações atuais da corrente do piano tangente" ou das reivindicações históricas por Marius ou por Schröter. De acordo com Francesco Mannucci, Fiorentino, podemos ler em seu diário (Fevereiro, 1711) que Cristofori tinha começado a trabalhar no novo cravo com piano e forte dois anos antes do centenário (o que significa 1698). ${ }^{18}$ Temos, também, uma data precisa encontrada em uma inscrição, ou melhor em uma nota manuscrita marginal, pelo músico de corte florentino Federigo Meccoli que em sua cópia do Le istitutioni harmoniche de Gioseffo Zarlino, indica juntamente com informações interessantes sobre como tocar o novo instrumento: Estas são as maneiras em que se é possivel tocar o Arpi Cimbalo de piano e forte. Inventado pelo Maestro Bartolomeo Cristofani (sic) de Pádua no ano de 1700, construtor de cravos do Sereníssimo Grão Príncipe Ferdinando da Toscana..$^{19}$ Note que Meccoli chamou o instrumento de Arpi Cimbalo, provavelmente da mesma maneira que ele ouviu do próprio Cristofori (G00D, 2005, p. 95-97).

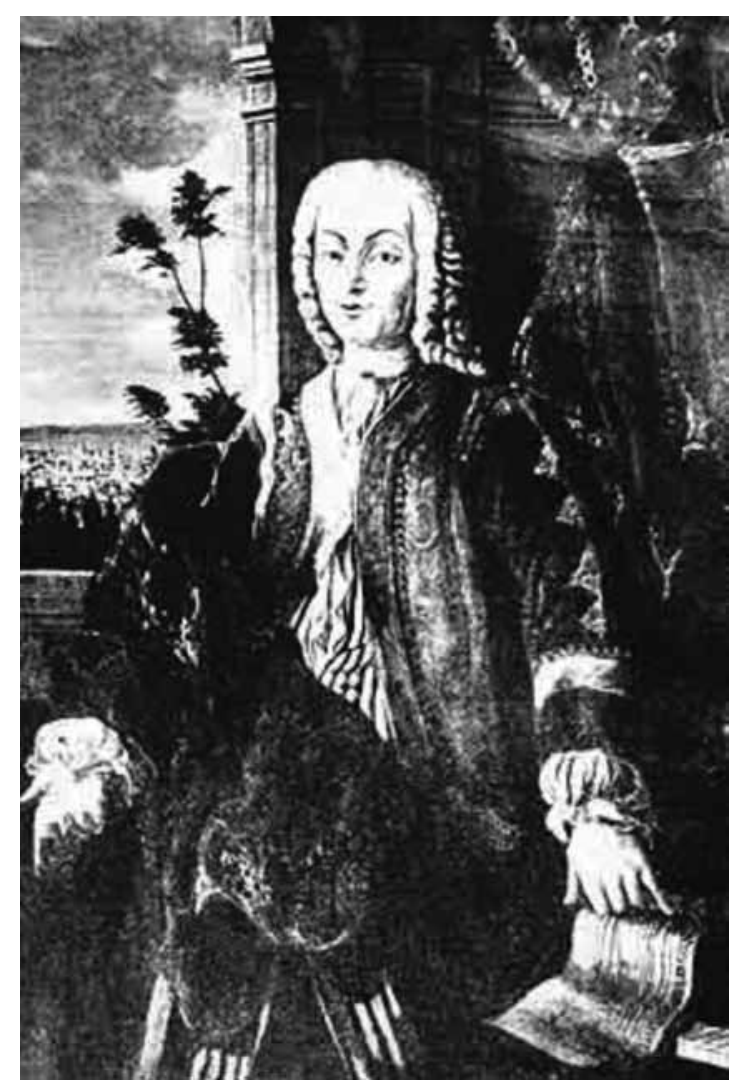

Ex.4 - Bartolomeo Cristofori.

(In:http://www.deutsches-museum.de/info/veranst/img/CristoforiBildnis.jpg) 


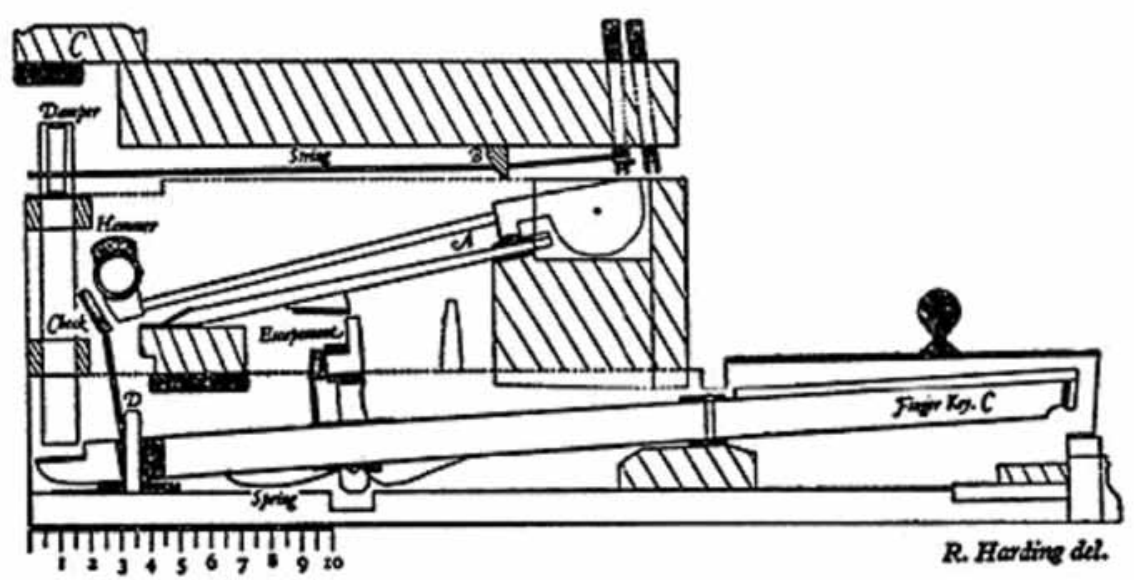

Ex.5 - Mecânica Cristofori (Harding, 1932).

In: http://www.metmuseum.org/toah/hd/cris/hd_cris.htm

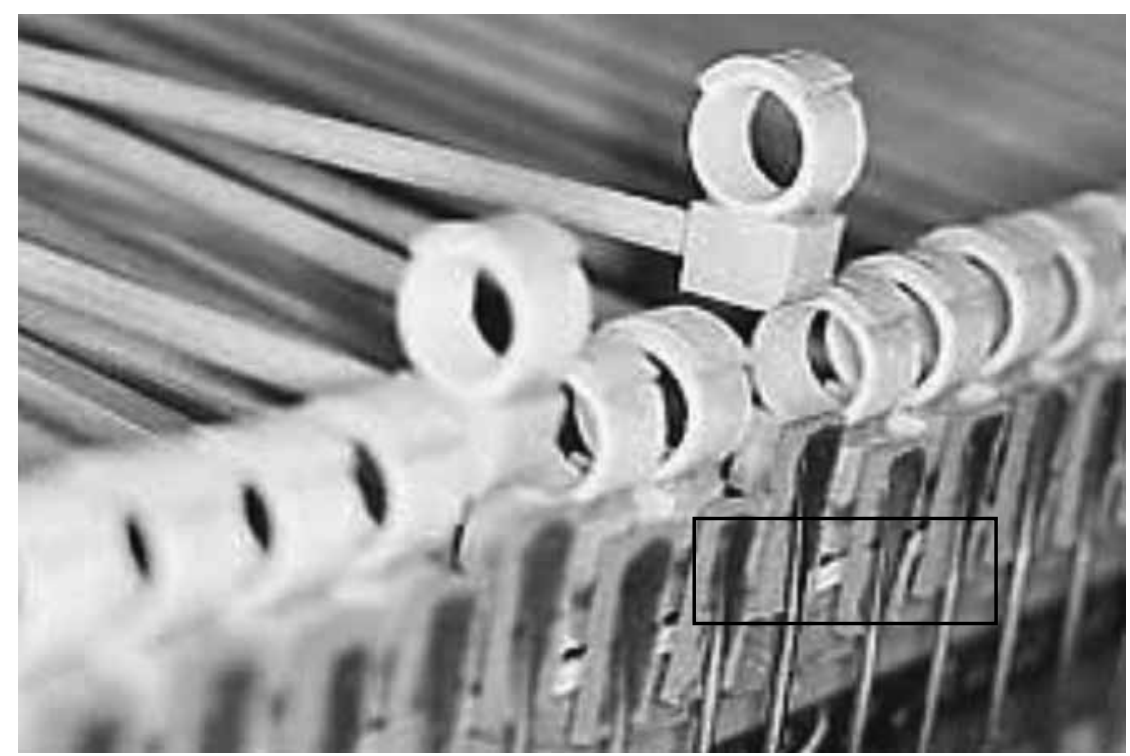

Ex.6 - Mecânica Cristofori com martelos empapelados por Denzil Wraight, Coelbe-Schoenstadt, Alemanha (In: http://www.denzilwraight.com/crisfp.htm).

Se o cravo pode ser considerado um desenvolvimento do saltério, o piano pode ser considerado um desenvolvimento dos dulce melos (dulcimer). Mesmo que, do incansável intelecto de Cristofori tenha vindo a idéia de uma mecânica com martelos antes de sua mudança para Florença, somente vivendo e trabalhando nessa cidade sob o patrocínio de Ferdinando, Cristofori pode ter êxito em desenvolvê-la. Na Itália parece ter havido pouco resultado imediato à monumental realização de Cristofori.

Externamente a construção do fortepiano de Cristofori parece similar a do cravo italiano do século XVIII. Na sua caixa ele introduziu um bentside (ou flanco curvo) interno para dar suporte ao tampo harmônico. 0 bentside interno permitiu que o tampo harmônico fosse estruturalmente isolado das seções críticas da caixa, o que permite um tampo harmônico especialmente ressonante.

0 erudito construtor de cravos e fortepianos Denzil Wraight (Ex.6), da Alemanha, escreveu a seguinte explanação em seu web site: ${ }^{20}$

A amplitude dinâmica desta reprodução (baseada nas mecânicas de 1722 e 1726 de Cristofori) é também maior do que se espera, graças, em parte, pela construção do bentside duplo de Cristofori. Este desenvolvimento notável na estrutura do cravo em dois frames (ou barragens internas) separadas, um para o tampo harmônico e outro para as cordas resolveu o problema de usar uma tensão mais elevada das cordas e de se evitar as conseqüências da distorção inevitável da caixa no tampo harmônico. Era tão adiante de seu tempo que só por volta dos anos 1820 os fabricantes de piano começaram a produzir os projetos estruturais que rivalizaram o de Cristofori na eficácia. 
A mecânica desenvolvida por Cristofori nos fortepianos de 1722 e 1726 é um pouco mais pesada que a mecânica vienense, ou Prellmechanik, dos anos 1790, o que é em parte explicado pela haste mais longa do martelo (Ex.7). Entretanto, uma mecânica leve não é, necessariamente, rápida. Nestas mecânicas desenvolvidas por Cristofori as teclas são um pouco mais curtas que em seus instrumentos anteriores a 1720 (cujo design foi a base do fortepiano português), muito mais curtos do que em mecânicas vienenses, mas tem um ponto diferente de equilibrio que faz o toque ligeiramente mais pesado e desse modo, rápido. Junto com o backcheck, que Cristofori também inventou, estas características do projeto produzem uma mecânica rápida e capaz de repetição eficiente e confiável.
Embora os pianos originais e reproduções do final do século XVIII tenham se tornado familiares ao intérprete moderno nos últimos anos, os instrumentos mais antigos tais como o de Cristofori, continuam a ser pouco familiares uma vez que os três instrumentos sobreviventes residem em museus e poucas reproduções tem sido feitas. ${ }^{21}$

Os três pianos de Cristofori que chegaram até nós são:

- 1720 - Metropolitan Museum of Art em New York City,

- 1722 - Collezione degli Strumenti Musicali em Roma,

- 1726 - Musikinstrumenten-Museum da Universidade de Leipzig (Ex.8).

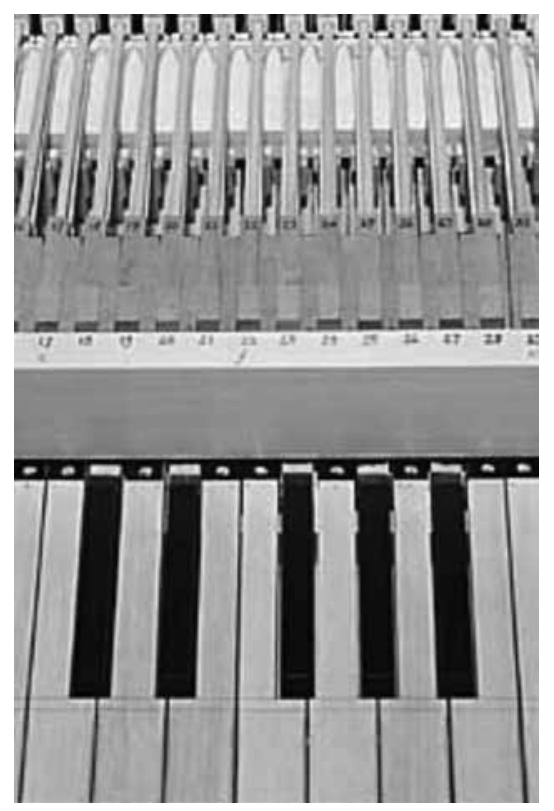

Ex.7 - Teclado e martelos de Denzil Wraight. Coelbe-Schoenstadt, Alemanha.

(In: http://www.denzilwraight.com/crisfp.htm).

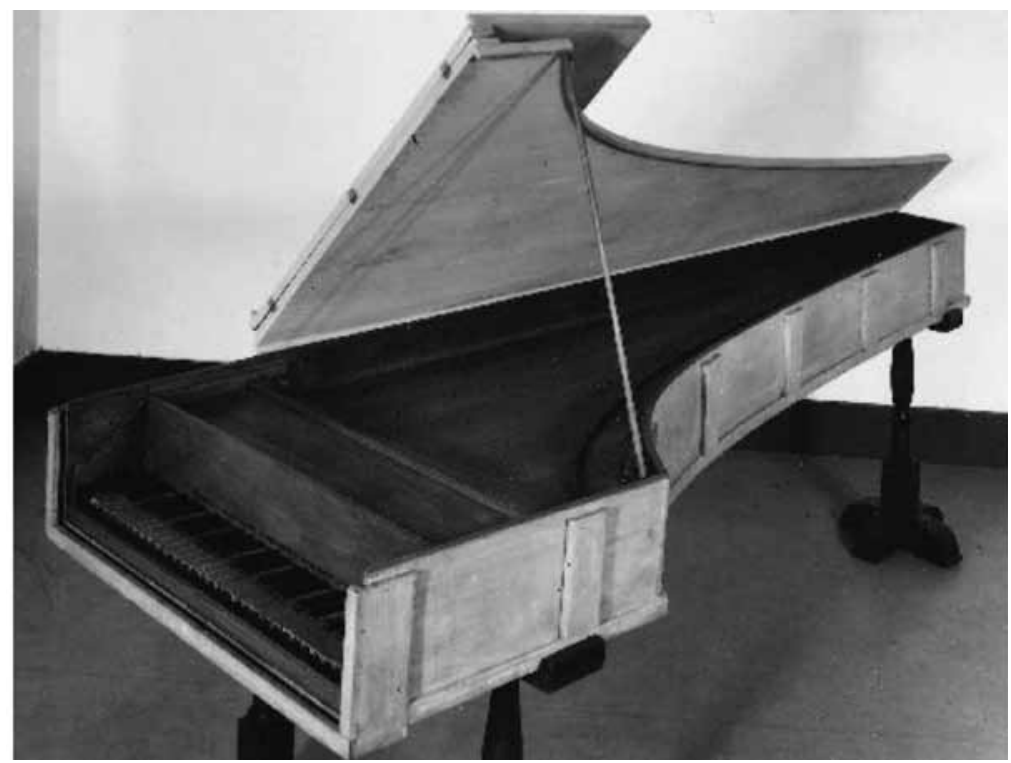

Ex.8. Piano de Bartolomeo Cristofori, Florença, 1726.

(Musikinstrumenten-Museum, University of Leipzig).

In: http://www.oxfordmusiconline.com/subscriber/article/img/grove/music 
0 Cristofori do Metropolitan, ou seja, seu mais antigo piano sobrevivente, tem uma caixa em forma tradicional, um único teclado e nenhum registro especial. Sua extensão ${ }^{22}$ vai, hoje, do C ao f'". Originalmente era do FF ao c'", sendo que o FF \# e GG \# eram omitidos. 0 instrumento tem as seguintes inscrições (POLLENS, 1984, 67):

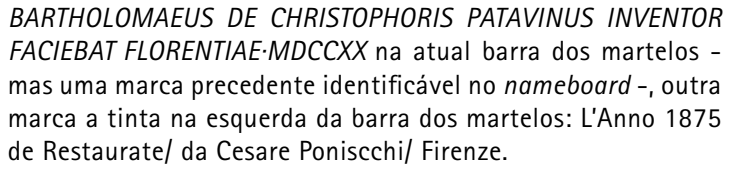
FACIEBAT FLORENTIAE.MDCCXX na atual barra dos martelos mas uma marca precedente identificável no nameboard -, outra marca a tinta na esquerda da barra dos martelos: L'Anno 1875 de Restaurate/ da Cesare Poniscchi/ Firenze.

A alteração envolveu elevar a nota inferior em uma quinta - o FF ao C, ou, de fato cinco teclas: FF, GG, AA, AA \#, $\mathrm{BB}$. Isto podia ser conseguido reduzindo o keyframe (ou base do teclado) trapezoidal no baixo para estendê-lo no agudo, deslocando-o à esquerda. 0 responsável pela reforma teve que fornecer uma nova medida movendo a ponte para mais perto da nut (ou pequena ponte sobre o cepo das cravelhas). Uma restauração maior ocorreu em 1938, já no Metropolitan Museum of Art. Curt Sachs (1881-1959) atuou como o consultor e sob seus conselhos o construtor do piano, Wolfgang Staub, empreendeu o trabalho. Desta vez peças importantes foram removidas para serem substituidas por outras novas. Infelizmente incluíram:

- Tampo harmônico,

- soundboard ribs (ou costelas sob o tampo harmônico),

- parte inferior do cepo das cravelhas

- nut (pequena ponte sobre o cepo das cravelhas),

- ponte

- fundo da caixa,

- cordas,

- cobertura dos martelos

- material do abafador.
Em 1970 o curador assistente de instrumentos musicais, Edwin Ripin, reencordoou o instrumento inteiramente em bronze, substituiu o couro no registro abafador, e reparou algumas partes da mecânica inadequadamente com cola epóxi branca! Desde então, ocorrem problemas com afinação, tendo por resultado ruptura de cordas. 0 que nos leva a considerar este fato como um indício para encordoar parcialmente com ferro.

Uma situação diferente pode ser encontrada nos dois outros pianos sobreviventes (1722 e 1726). Com uma extensão do C ao C'", o teclado desliza lateralmente, de modo que somente uma das duas cordas de cada nota seja golpeada, um verdadeiro registro una corda, reduzindo a sonoridade de todo o instrumento. Além deste efeito, Cristofori não fornece nenhum outro registro para alternar o som.

Pode-se imaginar que muito do trabalho feito em seus últimos instrumentos (1720-1731) tenha sido realizado por seu aprendiz e empregado, Giovanni Ferrini, uma vez que Cristofori morreu com a idade de 76 anos em 1731. Ferrini continuou a usar a mecânica de seu mestre, e as fortes similaridades podem ser vistas nos exemplares que sobreviveram de Cristofori e de Ferrini.

Cinco pianos de Cristofori e/ou de seu aluno Giovanni Ferrini foram comprados pela Rainha Maria Bárbara de Braganza da Espanha, patroa e discípula de Domenico Scarlatti (1685-1757). Centenas das mais de 550 sonatas para teclado de Scarlatti em movimento único podem ter sido pretendidas para o piano em lugar do cravo como se supõe há tanto tempo.

Scipione Maffei achou o piano mais apropriado para solos e para acompanhar a voz ou instrumentos solo, que grupos grandes devido a seu timbre intimista. Se comparado ao cravo, este possui um som mais brilhante e volumoso do que estes primeiros pianos.

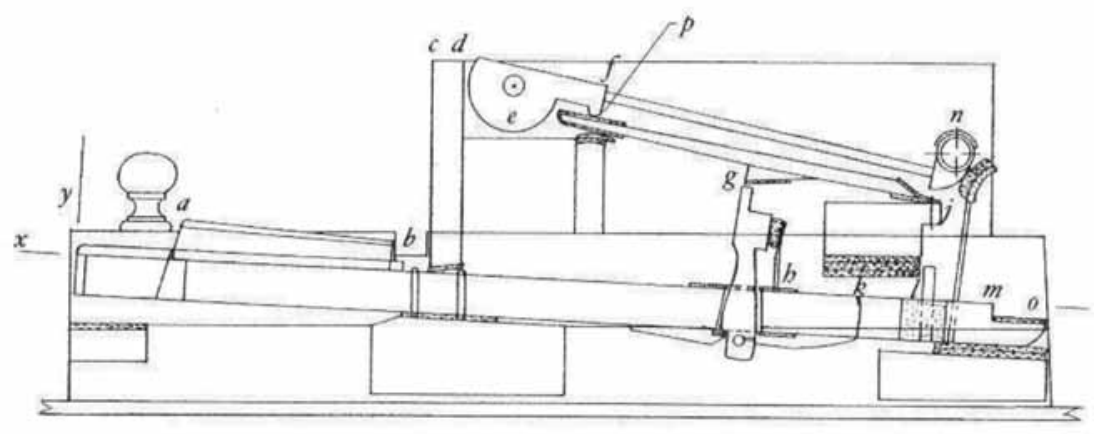

Ex.9 - Mecânica Silbermann.

In: SUTHERLAND, David A. Silbermann, Bach, and the Florentine piano. p.53. 


\section{3- A continuidade imediata}

Johann Ulrich König (MATTHESON, 1722-1725, 336343) traduziu o artigo de Maffei, incluindo o diagrama mecânico da Fig.5 acima. Johann Mattheson (16811764) incluiu a tradução mencionada em seu Critica Musica, parte ii publicado em 1725. Esta tradução deve ter sido a primeira fonte a chamar a atenção do famoso construtor de cravos e órgãos saxão, Gottfried Silbermann (1683-1753), para a grande realização de Cristofori. Silbermann iniciou suas experiências com a mecânica do fortepiano por volta de 1730 (Ex.9). Suas tentativas iniciais, não aprovadas por Johann Sebastian Bach (1685-1750), tinham um toque pesado e um agudo débil. Mais tarde, Bach elogiou os pianos de Silbermann e tornou-se agente de vendas para seus instrumentos (WOLFF, 2000, 412 e 427). Podemos acreditar, devido às similaridades, que Silbermann teve uma experiência real em primeira mão com um instrumento de Cristofori. ${ }^{23} \mathrm{As}$ melhorias subseqüentes fizeram dos pianos Silbermann particularmente bem sucedidos, levando Frederico, o Grande (1712-1786), rei da Prússia, a adquirir diversos exemplares. Silbermann manteve o cepo invertido de Cristofori, o espaçamento das cordas, os martelos ocos feitos de papeis enrolados (Ex.6 acima), encontrados no fortepiano Cristofori de 1726 e diversos outros detalhes. Silbermann, como Cristofori, mantém encordoamento duplo em toda a extensão.

Como ilustre representante da tradição de construção de instrumentos de teclado do norte europeu, Silbermann incluiu registros manuais para levantar os abafadores agudos e graves, além dos dispositivos de Cristofori para deslizar o teclado lateralmente de modo que os martelos golpeassem somente uma das duas cordas disponiveis para cada nota. Se as diversas variantes nas mecânicas de Cristofori nos mostram uma constante busca experimental rumo à evolução e melhoria, os exemplos de Silbemann são dimensionalmente muito consistentes e até mesmo conservadoras, com um elevado nivel artesanal e de refinamento. Embora Gottfried Silbermann e seu sobrinho Johann Heinrich Silbermann (1727-1799) fizessem cópias diretas da mecânica a martelos de Cristofori, outros fabricantes alemães, alguns deles não familiarizados à mecânica de Cristofori, planejavam mecânicas menos complicadas. Alguns deles adotaram a forma retangular do clavicórdio tendo por resultado os square-pianos. Em um dos primeiros exemplares, um martelo articulado à parte traseira da caixa é mantido ascendente por um bloco no fim da tecla, tendo por resultado uma simplificação grosseira da mecânica de Cristofori reduzindo-a a um mínimo. Este tipo de mecânica transformou-se na Stossmechanik, que poderia ser considerado como o princípio básico do que posteriormente viria a ser o piano inglês. A era de ouro da construção de piano no mundo saxão, entretanto, não será representada por nenhum destes desenvolvimentos ou mesmo por instrumentos de Silbermann, que, com a morte de seu filho, parece ter sido conduzido a um fim na linha direta de construção inspirada por Cristofori. Ao contrário, um novo conceito mecânico surgiu através de um aprendiz de Silbermann usando um principio mecânico que ficou conhecido como Prellmechanik - o qual dominaria a construção de pianos alemães/vienenses pelas sete décadas seguintes. Mas isto já será matéria para outros artigos.

\section{Referências Bibliográficas}

AUT VAR. Bologna, Civico museo bibliografico musicale, MS DD/53; Florence, Biblioteca del Conservatorio di musica Luigi Cherubini, MS D. 2534. $17^{\text {th }}$ Century Keyboard Music, v.10. New York: Garland, 1987.

BADURA-SKODA, Eva. "Prolegomena to History of Viennese Fortepiano". Israel Studies of Musicology, n.2, p.77-99, 1980.

BADURA-SKODA, Eva \&t Paul. Interpreting Mozart on the Piano. London: Barrie \& Rockliff, 1962.

BELT, Philipp R., Maribel Meisel, et. al. The Piano. New York: W. W. Norton, 1988.

BILSON, Malcolm. The Viennese Fortepiano of the Late $18^{\text {th }}$ Century. Early Music, v.8, n.2, p.158-162, April 1980.

BLOCH, Joseph. "Lodovico Giustini and the first published piano sonatas." The Piano Quarterly, 22, p.20-24, 1974.

BRUNELLI BONETTI, B. e A. Damarini. "Bartolomeo Cristofori e il mondo musicale padovano" in: Bartolomeo Cristofori, inventore del pianoforte, Publicazione ufficiale del comitato per la celebrazione del III centenario della nascità di Bartolomeo Cristofori. Padua: a cura dell' Ente provinciale per il Turismo, 1955.

CASELLI, Ala Botti. "Le 'Sonate da Cimbalo di Piano, e Forti' di Lodovico Giustini - L' Opera di un prete galante agli albori della sonata per pianoforte". Nuova Rivista Musicale Italiana, v.12, p. 34-66,1978.

CLINKSCALE, Martha Novak. Makers of the piano, 1700-1820, vol. 1. Oxford: Oxford University Press, 1993.

COLE, Michael. The Pianoforte in the Classical Era. Oxford: Oxford University Press, 1998.

DAVID, Hans Theodore \& Arthur Mendel. The Bach reader - A life of Johann Sebastian Bach in letters and documents. New York: W. W. Norton, 1972.

FABBRI, Mario. "La collezione medicea degli strumenti musicali in due sconosciuti inventari del primo seicento." Note 
d'Archivio per la Storia Musicale, supplemento 1, p.51-62, 1983.

"Nuova luce sull'attività fiorentina di Giacomo Antonio Perti, Bartolomeo Cristofori e Giorgio F. Haendel." Chigiana, v.21, p.143-90, 1964.

FANELLI, Jean Grundy. Giustini, Lodovico (Maria) in New Grove Dictionary, $2^{\text {nd }}$ Edition. London: Macmillan, 2001, v.IX, p.915. A chronology of Operas, oratorios, Operettas, Cantatas, and miscelaneous stage works with music performed in

Pistoia - 1606-1943. Bologna: Pendragon, 1998.

"La musica per la chiesa e l'oratorio di San Prospero nel Sei-Settecento." Bulletino Storico Pistoiese, v.XCII,

(Terza Serie, XXV), p.59-76, 1990.

"La musica patrocinata dai Rospigliosi: II Colegio dei Nobili." Bulletino Storico Pistoiese, v.XCVIII, (Terza Serie XXXI), p.113-28, 1996.

"The Manfredini family of musicians of Pistoia, 1684-1803." Studi Musicali, v.XXVI, n.1, p.187-232, 1997.

The Oratorios of Giovanni Carlo Maria Clari. Bologna: Pendragon, 1998.

FERGUSON, Howard. Keyboard Interpretation from Fourteenth to Nineteenth Century: An Introduction. London: Oxford University Press, 1979.

FREEMAN, Daniel E. "Lodovico Giustini and the emergence of the keyboard sonata in Italy." Anuario Musical, v.58, p.111-38, 2003.

FRUM, Bernard. An early example of dramatic procedures in $18^{\text {th }}$-century keyboard music. Musical Quarterly, v.56, p.230-46, 1970.

G00D, Edwin M. What did Cristofori call his invention? Early Music, v.33, n.1, p.95-7, February 2005.

HARDING, Rosamund Evelyn Mary. "The earliest pianoforte music". Music and Letters, v.13, p.194-9, 1932.

HUBER, Alfons. Was the 'Viennese Action' Originally a Stossmechanik? The Galpin Society Journal, v.55, p.169-182, April 2002.

KIRBY, Frank E. A short history of keyboard music. New York: Free Press, 1966.

KOMLÓS, Katalin. Fortepianos and their Music. Oxford: Oxford University Press, 1995.

LATCHAM, Michael. Franz Jakob Spath and the Tangentenflüegel, an Eighteenth-Century Tradition. The Galpin Society Journal, v.57, p.150-70, May 2004.

The check in some early pianos and the development of piano technique around the turn of the $18^{\text {th }}$ century.

Early Music, v.21, n.1, p.28-42. February 1993.

Mozart and the Pianos of Johann Andreas Stein. The Galpin Society Journal, v.51, July 1998, p.114-153.

Swirling from one level of the affect to another: the expressive Clavier in Mozart's time. Early Music, v.30, n.4, p.502-520. November 2002.

MAFFEI, Scipione (1675-1755). "Nuova invenzione d'un gravicemablo col piano e forte." Giornale de'letterati d'Italia, v.V (Venezia, 1711), p.144-59.

MARSHALL, Robert Lewis. Eighteenth-Century Keyboard Music. New York: Schirmer, 1994.

MATTHESON, Johann (1681-1764). Critica Musica (Pars I-VIII. 1722-1725). Amsterdam: Frits Knuf, 1964.

MOBBS, Kenneth. A Performer's Comparative Study of Touchweight, Key-dip, Keyboard Design and Repetition in Early Grand Pianos, c. 1770 to 1850. The Galpin Society Journal, v.59, p.16-44, May 2001.

MONTANARI, Giuliana. Bartolomeo Cristofori - A list and historical survey of his instruments. Early Music, v.19, n.3, p.383-396, August 1991.

O'BRIEN, Michael Kent. Bartolomeo Cristofori at court in late Medici Florence. Dissertation, Washington D.C.: The Catholic University of America, 1994.

OORT, Bart van. Haydn and the English Classical Piano Style. Early Music, v.28, n.1, p.73-89, February 2000.

PARAKILAS, James. Piano roles: three hundred years of life with the piano. New Haven, CT: Yale University Press, 1999.

PEREIRA, Mayra. Do cravo ao pianoforte no Rio de Janeiro: Um estudo documental e organológico. Dissertação de Mestrado. Rio de Janeiro: Universidade Federal do Rio de Janeiro, 2005.

PERSONE, Pedro. The earliest piano music: Lodovico Giustini's (1685-1743) Sonate da cimbalo di piano, e forte ditto volgarmente di martelletti, Firenze, 1732. Dissertação de Doutorado. Boston: Boston University, 2006.

The earliest piano music: Lodovico Giustini's (1685-1743) Sonate da cimbalo di piano, e forte ditto volgarmente di martelletti, Firenze, 1732. Saarbrücken: VDM-Verlag, 2008.

PINESCHI, Umberto. "Jacopo Melani e Ludovico Giustini, organisti della Congregazione dello Spirito Santo di Pistoia." Bullettino Storico Pistoiese, v.LXXV, p. 89-94, 1973.

POLLENS, Stewart. The Early Pianoforte. Cambridge: Cambridge University Press, 1995.

The Bonafis spinet: an early harpsichord converted into a tangent piano. Journal of the American Musical

Instrument Society, v.11, p.5-21, 1985.

Christoph Gottlieb Schröter, inventor or fraud? Early Keyboard Journal, v.18, p.139-53, 2000.

The early Portuguese piano. Early Music, v.13, n.1, p.18-27, February 1985. 
The Gatti-Kraus piano action ascribed to Bartolomeo Cristofori. Galpin Society Journal, v.40, p.269-78, April 2002. Gottfried Silbermann's pianos. Organ Yearbook, v.17, p.103-21,1986.

The pianos of Bartolomeo Cristofori. Journal of the American Musical Instruments Society, v.10, p.32-68, 1984.

. Three keyboard instruments signed by Cristofori's assistant Giovanni Ferrini. Galpin Society Journal, v.44, p.77-93, March 1991.

POWERS, Wendy. "The Pianofortes of Bartolomeo Cristofori (1655-1731)". In: Heilbrunn Timeline of Art History. New York:

The Metropolitan Museum of Art, 2000-. http://www.metmuseum.org/toah/hd/cris/hd_cris.htm (October 2003)

RATTALINO, Piero. Storia del pianoforte: lo strumento, la musica, gli interpreti. Milano: Saggiatore, 1983.

ROSENBLUM, Sandra P. Performance Practice in Classic Piano Music. Bloomington, IN: Indiana University Press, 1988.

ROWLAND, David. Early Keyboard Instruments - A Practical Guide. Cambridge: Cambridge University Press, 2001.

RUSSEL, Raymond. The harpsichord and the clavichord, $2^{\text {nd }}$ edition. New York: W. W. Norton, 1973.

SCHOT, Howard. From the harpsichord to pianoforte: a chronology and commentary. Early Music, v.13, n.1, p.28-38, February 1985.

SELFRIDGE-FIELD, Eleanor. The invention of the fortepiano as intellectual history. Early Music, v.33, n.1, p.81-94, February 2005.

SHEVELOFF, Joel Leonard. Domenico Scarlatti: Tercentenary Frustrations. Musical Quarterly, v.71, p.399-436, 1985.

. Domenico Scarlatti: Tercentenary Frustrations, part II. Musical Quarterly, v.72, p.90-118, 1986.

SILVESTRI, Giuseppe. Scipione Maffei (1675-1755) - Europeo del Settecento. Vicenza: Neri Pozza, 1968.

STEVENSON, Robert. Some Portuguese sources for early Brazilian music history. Inter-American Institute for Musical Research, v.4, p.1-43, 1968.

The Brazilian Benedictine Bishop who launched the first piano publication (1732). Inter-American Music Review, v.1, p.211-15, 1978/79.

SUTCLIFFE, W. Dean. The Keyboard Sonatas of Domenico Scarlatti and the Eighteenth-Century Musical Style. Cambridge: Cambridge University Press, 2003.

SUTHERLAND, David A. Domenico Scarlatti and the Florentine piano. Early Music, v.33, n.2, p.243-256, May 1995. . Silberman, Bach, and the Florentine piano. Early Keyboard Journal, v.21, p.45-63, 2003.

TAGLIAVINI, Luigi Ferdinando. Giovanni Ferrini and his harpsichord 'a penne e a martelletti'. Early Music, v19, n.3, p.399408, August 1991.

WOLFF, Christoph. Johann Sebastian Bach, the learned musician. New York: W.W. Norton, 2000.

\section{Notas}

1 Estas foram as qualidades utilizadas por Johann Andreas Stein (1728-1792) para dizer a Mozart sobre as qualidades do fortepiano. Vide carta de Mozart a seu pai datando de 17 de Outubro de 1777.

2 Este desenho me foi enviado via fax por G. Potvlieghe como suporte para minha explanação sobre a evolução do fortepiano. Por este motivo não consta das referencias bibliográficas.

3 Até então o dulcimer era tocado, principalmente, em tavernas ou locais semelhantes.

4 Usado pela primeira vez por Silbermann.

5 Adi 6. Mag.o 1655 Bartolomio F.o di Fran.co Christofani et di Laura sua consorte fù battezato da me D.on Gagliardi suo parocho com.e al s. Fonte il s. Camillo Chinoni della parochia di s. Lorenzo. Comadre fù Pani Lina serva della sg.ra Laura Papafava della parochia del Duomo nato adi 4 corrente hore 6 c.a.

6 Cembalaro é a palavra italiana para construtor de cravos.

7 Che fu detto al Principe, che non volevo; rispos'egli il farò volere io... " Foi dito ao Príncipe que não desejava (me juntar à sua corte) ao que ele respondeu que me faria desejar".

8 Infelizmente nenhum instrumento de seu período de Pádua chegou até nós.

9 Registros de despesas do Príncipe Ferdinando estabelecem a data da contratação de Cristofori como sendo 1688, bem como sua equiparação com os músicos da corte.

10 Projeto que começou ca. 1698 , após algumas idéias que trouxe de Pádua.

11 Inventário de diversos tipos de instrumentos musicais de propriedade do Sereníssimo Príncipe Ferdinando da Toscana, 1700.

12 Un Arpicembalo di Bartolomeo Cristofori di nuova invenzione, che fa' il piano, e il forte, a' due registri principali unisoni, com fondo de cipresso senza rosa, con fascie e scorniciatura mezza tonda simile, con filetto d'ebano, con alcuni saltarelli con pano rosso, che toccano nelle corde, et alcuni martelli, cha fano il piano, et il forte, e tutto l'ordingo vien serrato, e coperto da un piano di cipresso filettato di ebano, con tastatura di bossolo, et ebano senza spezzati, che comincia in cisolfaut ottava stesa, e finisce in cisolfaut, con n. quaranta

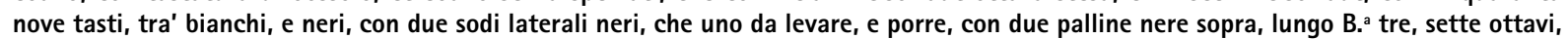
largo nel davanti B. ${ }^{a}$ uno, e soldi sei con suo leggio di cipresso, e sua contro cassa d'albero bianca, e sua coperta di cuoio rosso foderata di taffettà verde e orlata di nastrino d'oro.

13 Verona, Biblioteca Capitolare DCCCLX, fasc. VI, no. 1, Studii Miei.

14 Maffei, Scipione. Nuova invenzione d'un gravicembalo col piano e forte. Giornale de'letterati d'Italia, vol. V (Venezia, 1711), p.144-159.

15 Bortolo Critofali padovano stipendiato das S. Principe ha inventato senza motivo avuto de altra cosa il cimbalo col piano e forte, ne ha fatto tre fin ora, due venduti in Firenze, uno al Card. Ottoboni. 
16 Cardeal Otoboni é também conhecido por hospedar a famosa perfidia entre Handel e Scarlatti. Esse encontro-desafio fez uso de diferentes instrumentos e muito provavelmente o Arpicembalo estava entre eles.

17 Bartolomeo Cristofori, foi apontado para a posição de curador em 1716 por ordem do Grande Duque Cosimo III, supervisionando o inventário de instrumentos musicais nas propriedades de Ferdinando.

18 Nuovi Cimbali con piano e forte, fabricatti nel Laburatorio per volere del Ser.mo Gran Principe Ferdinando, cominciato du'anni prima del Giubileo.

19 Questi sono gl'andamenti che si possono adattare in su I' Arpi cimbalo del piano e forte, inventato da M.ro Bartolomeo Cristofani Padovano. L'Anno 1700. Cimbalaro del Ser.mo Gran P.pe Ferdinando di Toscana. Luisa Cervelli. Noterelle Cristoforiane. Quadrivium, 22, 1,1981, p.157.

20 Idem.

21 Podemos citar os seguintes construtores que pesquisam e copiam pianos segundo Cristofori: Denzil Wraight (Alemanha), Kerstin Schwarz (Itália), David Sutherland (USA), Thomas and Barbara Wolf (USA), Keith Hill (USA) e Nobuo Yamamoto (Japão).

22 Utilizo a nomenclatura de Hermann Ludwig Ferdinand von Helmholtz (1821-1894).

23 Segundo pesquisas e correspondências entre Denzil Wraight e David Sutherland deve ter sido um instrumento Ferrini. Entre os pesquisadores somente Wolf Dieter Neupert acredita que Silbermann trabalhou exclusivamente com plantas ou desenhos e não com um instrumento real. A opinião de Neupert pode ser estudada no artigo "Gottfried Silbermanns Hammerflügel: eine Kopie Bartolomeo Cristoforis?", Jahrbuch des Staatlichen Instituts für Musikforschung Preußischer Kulturbesitz 2004, p.136-144.

Pedro Persone é Doutor em Música - D.M.A. - em performance histórica pela Boston University com bolsa de estudos da Capes e sua tese é: The Earliest Piano Music: Lodovico Giustini's (1685-1743) Sonate da cimbalo di piano e forte detto volgarmente di martelletti, Firenze, 1732, recentemente lançada em formato de livro pela VDM-Verlag de Saarbrücken, Alemanha. Mestre em Cravo pela UNICAMP sob orientação de Helena Jank (com pesquisa sobre obras non mesurés; com bolsa FAPESP). Na Europa, estudou com Huguette Dreyfus (École Nationale de Musique de Bobigny, França), cravo e fortepiano com Jacques Ogg (Academie voor Oude Muziek, Amsterdam) e com Patrick Cohen (França; bolsa da Fundação Vitae). Em 1991, foi o primeiro a reintroduzir o fortepiano no circuito musical brasileiro. De 2001 a 2004, foi professor de group piano na Boston University (EUA). Atualmente, realiza pós-doutorado com bolsa FAPESP sobre o tema $A$ música pianística e camerística composta entre 1790 e 1826 presente na 'Coleção Theresa Christina Maria': uma abordagem segundo as práticas interpretativas relacionadas ao período. 